\title{
THE BENEFITS OF A NETWORK TASKING ORDER IN COMBAT SEARCH AND RESCUE MISSIONS
}

\author{
Murat Gocmen, Kenneth Hopkinson, and Matthew Compton \\ Air Force Institute of Technology \\ Wright Patterson AFB, OH
}

\begin{abstract}
Networked communications play a crucial role in United States Armed Forces operations. As the military moves towards more network centric (Net-Centric) operations, it becomes increasingly important to use the network as effectively as possible with respect to the overall mission. This article advocates the use of a Network Tasking Order (NTO), which allows operators to reason about the network based on asset movement, capabilities, and communication requirements. The NTO is similar to the Air Tasking Order, which gives insight into the plan for physical assets in a military mission. In this paper we illustrate the benefit of an NTO in a Combat Search and Rescue (CSAR) scenario. While demonstrating the CSAR mission, we assume the use of the Joint Tactical Radio System (JTRS) for communication. Our premise is that the knowledge in an NTO can be used to achieve better CSAR missions and yield better decision-making opportunities to the mission commanders. Our results show that the End-toEnd (ETE) delay with the aid of an NTO in high traffic conditions is shorter compared to those without the NTO and bandwidth requirements are also lower. In low traffic conditions, the ETE delay is shorter without the aid of an NTO, but at the cost of higher bandwidth utilization.
\end{abstract}

\section{INTRODUCTION}

As networks continue to evolve to operate in wireless domains, future wars will move to net-centric warfare or operations. When net-centric operations are implemented, decision-makers will know more about battlefield situations. In this paper we show the benefits of a Network Tasking Order (NTO), which gives decision-makers a better big picture of the battlefield, through a simulated Combat Search and Rescue (CSAR) operation.

An NTO can be built by looking at the Air Tasking Order (ATO), which contains daily schedules of air related

The views expressed in this document are those of the authors and do not reflect the official policy or position of the United States Air Force, Department of Defense, or the U.S. Government. operations. The ATO includes details of a day's flights such as mission types, aircraft types, the number of aircraft, etc. The ATO is in official use in NATO. However, an NTO, which shows the network connectivity among the daily flights in the ATO, is still a theoretical construct.

The objective of CSAR operations is to protect downed/isolated personnel and reduce their threat level to allow recovery vehicles to pick them up. CSAR missions can be divided into two categories [2]. The first involves missions that are immediate; survivors must be rapidly recovered after they are downed. The second category of missions is deliberate; an assessment of mission considerations may require delay for more detailed planning. This paper focuses on deliberate CSAR missions.

The use of an NTO to aid in CSAR missions is illustrated using OPNET network simulations. CSAR forces will execute the same scenario under two different networking capacities by using Joint Tactical Radio System (JTRS) as a communication device. The first scenario is implemented without using the NTO as a guide. The second scenario is implemented using the NTO. The results show real potential for the use of the NTO in CSAR missions involving deliberative planning. Summaries of the CSAR mission, JTRS and the NTO follow.

\section{COMBAT SEARCH AND RESCUE}

CSAR operations have four phases: Search, Ingress, Execution and Egress. These phases start after the downed/isolated personnel are reported to the CSAR Joint Force Commander (JFC). The JFC will establish a CSAR Task Force (CSARTF) [1]. The CSARTF, illustrated in Figure 1, generally consists of Rescue Escort (RESCORT) vehicles, Rescue Combat Air Patrol (RESCAP) vehicles, Survivor(s), and an Air Mission Commander (AMC). RESCORT vehicles escort the recovery vehicles. RESCAP vehicles cross the Forward Line of Own Troops (FLOT), the most forward position of friendly forces at a specific time, to establish air superiority over the operation area and the ingress route. The Airborne Warning And Control System (AWACS) plays a mission monitoring role. 


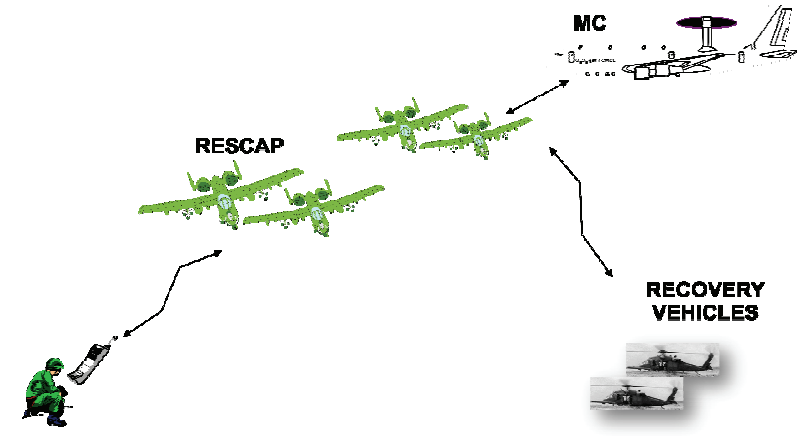

Figure 1. Roles of parties involved in CSAR missions

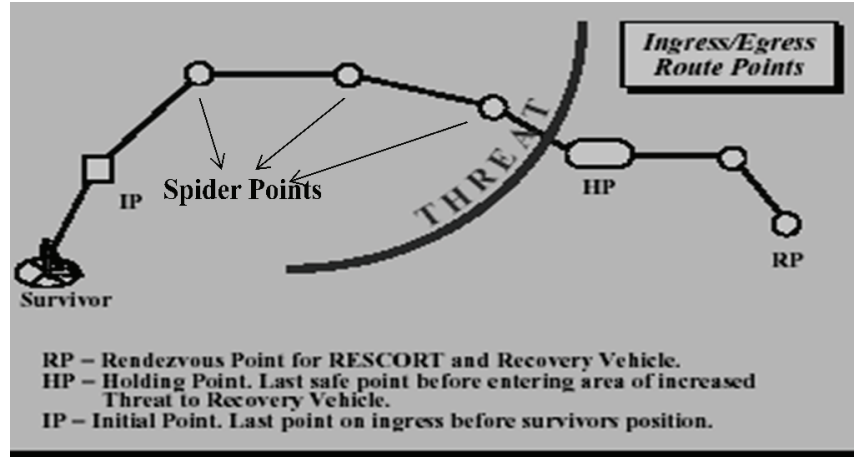

Figure 2. Interaction of RESCORT and RESCAP vehicles via Spider Points [1]

In order to see the benefit of the NTO in this research, we have to look the four phases of CSAR missions.

\section{SEARCH PHASE}

The aim of this phase is to find the rough location of the survivor(s). Usually this is done through one or more of the following means:

a. Position reported by wingman

b. Activation of aircraft emergency beacon

c. Survivor radio contact

d. Last known position from AWACS.

\section{INGRESS PHASE}

After the survivor(s) approximate position has been determined, all of the CSARTF assets will check-in with AMC [1]. RESCAP vehicles will cross the FLOT and gain local air superiority over the Operation Area and ingress route. RESCORT vehicles and recovery vehicles will be launched and repositioned in a holding area close to FLOT where the handover will take place between RESCORT and RESCAP vehicles [1].

\section{EXECUTION PHASE:}

This phase begins by finding the survivor(s). Once the survivor(s) has been precisely located and mission is considered feasible by the RESCAP vehicles after threat assessment has been completed, the RESCORT vehicles hand over the recovery vehicles to RESCAP vehicles at the
Holding Point (HP) [1]. The RESCAP vehicles will lead the recovery vehicles using Spider Points (SP) to the survivor. This is shown in Figure 2.

All assets should be aware that this is the most critical part of the mission, and therefore, they should minimize radio transmission. With that understanding, in this part of the mission there will be regular radio transmissions between the survivors, RESCAP, and the recovery vehicles. Also, the AMC should be ready to copy the radio transmissions and to relay them to CSARTF if line of sight communication between the RESCAP vehicles and recovery vehicles is not possible [1].

\section{EGRESS PHASE:}

After the survivor is evacuated, RESCAP and recovery vehicles will follow the Spider Points that are more secure, as was determined during the INGRESS phase. The Spider Points used could be same ones employed in the INGRESS phase or they could be different. The handover will take place at the HP. This is shown in Figure 2.

\section{JOINT TACTICAL RADIO SYSTEM}

JTRS is the next-generation software defined radio for use by the U.S. military in field operations after 2010 [3]. JTRS creates a family of radios for troops, vehicles, ships, etc. that share an underlying architecture, can use the Internet Protocol for data, and is a "software-defined" platform that relies on software rather than hardware to handle communication protocols [4]. JTRS will work with many existing radio standards. JTRS is intended to permit the Services to interoperate seamlessly via wireless voice, video, and data through all command levels, including direct access to near real-time information from airborne and battlefield sensors [4]. It includes integrated encryption and Wideband Networking Software to create mobile adhoc networks (MANETs). JTRS has been described as the "backbone" of the U.S. Army's Future Combat System [5]. The JTRS program is broken into five clusters, shown in Table 1, each with a particular Service "lead."

Despite some programmatic and technical issues, the JTRS Program continues to be a key DoD transformational program. The JTRS Program provides foundational support for the DoD objective of information superiority on the battlefield, meeting the growing demand of Warfighters' communications needs [5].

\section{NETWORK TASKING ORDER}

The ATO is a daily schedule of all air operations. The ATO contains the types and numbers of aircraft, the 
Table 1. The JTRS program's cluster

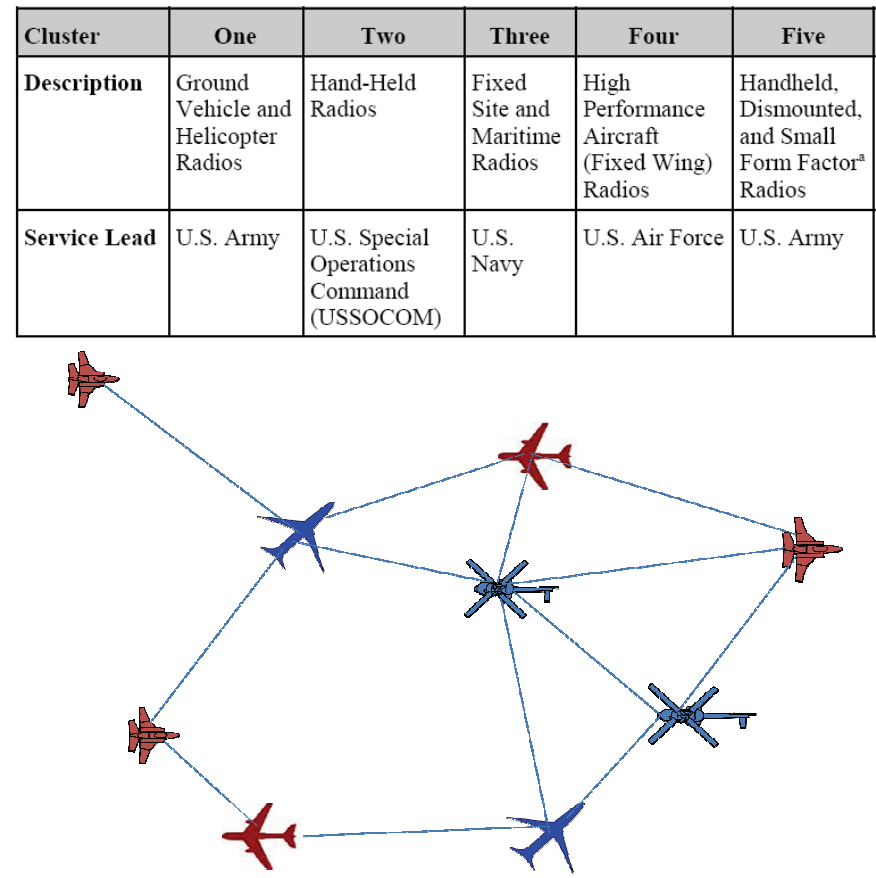

Figure 3. NTO Network Illustration

aircrafts' mission types and routes, communication channels, altitude, target positions, etc. We can easily define potential mission network communication by looking at the air vehicles' routes, their altitude, and the time frames of their presence in the ATO. This type of data, combined with auxiliary information, enables the idea of a Network Tasking Order (NTO). The word NTO is new, but the idea of an NTO is not new to the DOD. The feasibility of managing networking connections between the air vehicles has been investigated by many DOD entities. However, the first appearance of the term NTO, with the meaning just been presented, was in a group research project (GRP) at the Air Force Institute of Technology [6]. Follow-on work to the initial GRP has been performed by Matthew Compton and has appeared in a published article in the Military Communications Conference in 2008 [7].

Our aim is to show the benefits of an NTO in CSAR missions using the JTRS software-defined radio system through two experimental scenarios. CSAR is used to illustrate the utility of an NTO because CSAR is one of the most critical missions, from a communications perspective, amongst the major military mission types. In almost every CSAR phase, communication must be maintained for every asset. A second goal of this article is to present a vision of what future battles will look like when almost $100 \%$ network reliability is achieved at the battlefield.

The JTRS standard will go a long way towards solving interoperability communication problems for the US
Armed Forces. From the Air Force perspective, every asset in the ATO will communicate with each other. This will aid in the creation of the NTO. With the help of the NTO, network coverage will be seen as more critical and also seen by the decision-makers. An illustration of the network coverage afforded with an NTO is shown in Figure 3.

JTRS will define integrated encryption and an underlying architecture, which could use the Internet Protocol for data. For that reason the NTO will not be concerned with the interoperability of the assets' communication.

An NTO should contain the following information:

- Each asset's transmission range/speed

- The mission priority of the assets' communications

- The pre-planned routes through which the assets' packets will go.

Here is a sample of what an ATO looks like:

1- TSKCNTRY/US//

2-SRCVTASK/F//

3-TASKUNIT/555FS/ICAO:ETAD//

4-AMSNDAT/C2342/CSAR//

/DE/TGT-ID/LOCATION/TOT

/01/-/294248N0473106E/241200ZJAN/

/02/-/294300N0473896E/241215ZJAN/

/03/-/294300N0473805E /241233ZJAN/

/04/-/294236N0473106E /241303ZJAN//

5-MSNACFT/1/ACTYP:F16C/SANDY01/2MK-82

/1654/3322//

6-AMSNLOC/AGL200/1//

In the sample ATO above, the mission is divided into 6 lines. The first line represents the task country. In this case, the country is the U.S. The second line lists the service tasked, here the Air Force. The third line is the tasked unit, which is the 555 fighter squadron at Spangdahlem Air Base (ETAD). The fourth line gives the aircraft mission data. Here the mission number is $\mathrm{C} 2342$ and the mission type is Combat Search and Rescue. The following lines show the spider points coordinates $\left(29^{\circ} 42^{\prime} 48^{\prime \prime}\right.$ North $47^{\circ}$ $31^{\prime} 06^{\prime \prime}$ East), date of the flight (24 JAN), and times the aircraft will be over the spider point $(1200 \mathrm{Z})$. The fifth line shows the aircraft tasked for the mission. There is one F-16C with the call sign SANDY01. The aircraft is equipped with two MK-82 missiles. 1654/30111 are the Identification, Friend or Foe/Selective Identification Feature (IFF/SIF) codes. The sixth line is the altitude (200 feet above ground level) and priority (1) of the flight.

We can use the ATO to derive the NTO. An NTO derived from the ATO above could contain these lines: 


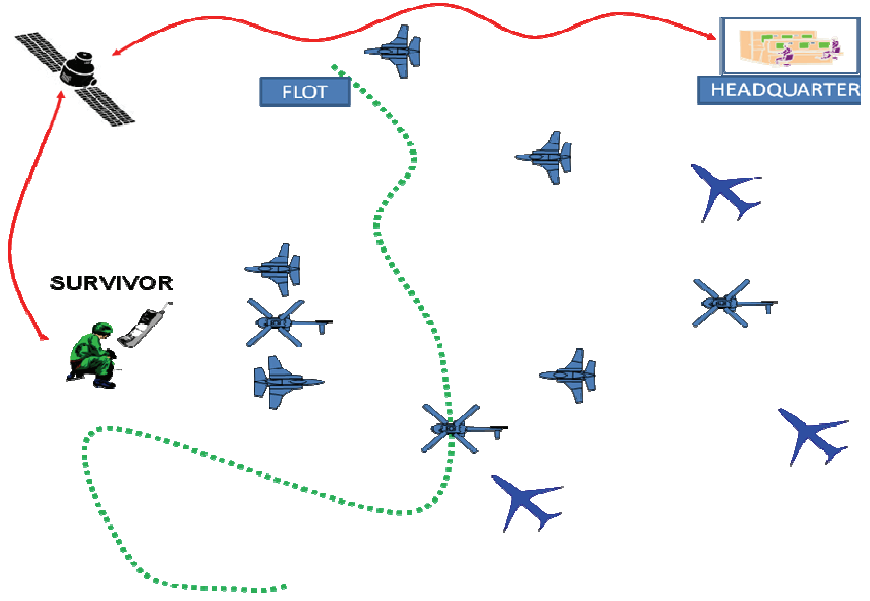

Figure 4. Snapshot of Air space

TSKNODE/US/F/555FS//

NODETYP/50/11MBPS//

PCKTPRIORTY/1//

PREPLANNEDRT/1652/3035/2342/1000//

The first line represents the country, service, and unit. The second line represents the asset's transmission range (50 miles) and speed (11 Mbps). The third line represents the packet priority. The fourth line represents preplanned routes that the packets will go through. The numbers (1652, 3035) represent the aircrafts' IFF codes.

\section{SIMULATION}

\section{Scenario:}

A CSAR scenario has been created using the OPNET Simulation MODELER (14.0) by adapting phases of the CSAR mission described earlier. Only the CSAR mission's execution phase is simulated since an entire CSAR mission can take a long period of time.

The CSAR execution phase is simulated under two different scenarios. These scenarios illustrate the NTO's benefits in the context of CSAR missions. Both scenarios reflect the dynamics of a CSAR mission using JTRS.

In the scenarios run, the downed pilot is first located by a satellite. Second, an image of the location and coordinates of the downed pilot are sent to the headquarters. This same data is sent to the recovery vehicle using two methods (one per scenario).

The overall architecture is shown in Figure 4. The solid line shows the data (location and image) flow from the survivor to the Headquarters. In the first scenario, the data is sent to all nodes in the network to reach the survivor. This is what we call the "CSAR without NTO knowledge" scenario. In the second scenario, the data is sent to specific nodes that were determined using the NTO in order to reach to the survivor. This is called the "CSAR with NTO knowledge" scenario.

There are two hypotheses considered in the simulations.

- The end-to-end delay will be longer for CSAR without NTO knowledge when compared to CSAR with NTO knowledge in high traffic situations.

- The end-to-end delay will be longer with the CSAR without NTO knowledge when compared to CSAR with NTO knowledge in low traffic situations.

In this study, the baseline of the two scenarios will be the ones without NTO knowledge. The comparison will try to search if any of the NTO knowledge scenarios does have a significant impact on the End-to-End (ETE) delay.

The simulation environment is solely designed for this simulation. Therefore a detailed description of the original simulation environment is explained thoroughly below.

While flying over enemy region, one of two aircraft is downed by a hostile forces Surface to Air Missile. The pilot landed safely on the ground, but he had some injuries and he cannot move. Since he cannot move, his exact position is very important to the CSARTF and the reaction time must be fast. The pilot's position is in a deep wadi (valley) so that, other than using a satellite system, it is not possible to detect the downed pilot. The downed personnel sends a beacon signal and this is located by a national satellite. Likewise, the location of the survivor and an image of the location is taken by satellite and sent to the headquarters. As soon as the downed aircraft is reported to CSAR JFC, CSARTF is deployed for the mission without knowing the exact location. The Headquarters is trying to send the exact location to CSAR mission assets so that CSARTF can accomplish the mission safely and efficiently.

In the first scenario, the data sent by the headquarters to the AWACS is broadcast to the recovery vehicle through every asset in the ATO. In the second scenario, flooding along four different routes designated by the NTO is simulated. The various routes are depicted in Figure 5. Route 1 floods all network routes.

The fixed experimental parameters are displayed in Table 2. These experiments attempt to verify the impact of the NTO on ETE delay. Table 3 summarizes the packet delay that results from scenarios 1 and 2. In scenarios 1 and 2, during each simulation run, all fixed parameter values are held constant. The packet inter-arrival time is 0.0625 seconds for scenario $1,0.1667$ seconds for scenario 2 , and 


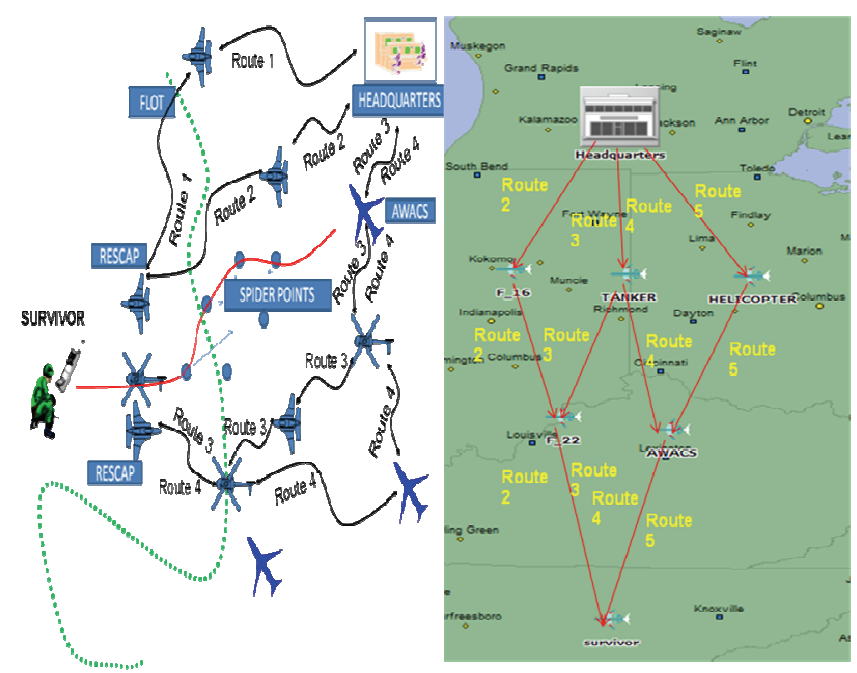

Figure 5. Scenario 1 with high traffic load

Table 2. Fixed Parameters

\begin{tabular}{|l|l|l|}
\hline Parameter & Scenario 1 Values & Scenario 2 Values \\
\hline Data Rate & $90,000 \mathrm{bps}$ & $90,000 \mathrm{bps}$ \\
\hline Packet Size, Distribution & $5000 \mathrm{bits}$, Constant & $5000 \mathrm{bits}$, Constant \\
\hline Number of Aircraft & 6 & 6 \\
\hline Channel Bandwidth & $20 \mathrm{kHz}$ & $20 \mathrm{kHz}$ \\
\hline Channel Min. Freq. & $400 \mathrm{MHz}$ & $400 \mathrm{MHz}$ \\
\hline Aircraft Ant. Power & $15 \mathrm{Watt}$ & $15 \mathrm{Watt}$ \\
\hline Simulation Length & $\begin{array}{l}2750 \mathrm{time} \text { steps } \\
(45 \mathrm{~min})\end{array}$ & $\begin{array}{l}2750 \text { time steps } \\
(45 \mathrm{~min})\end{array}$ \\
\hline
\end{tabular}

Table 3. Factor levels for Scenario 1 and Scenario 2

\begin{tabular}{|c|c|c|}
\hline Factor & Level 1 & Level 2 \\
\hline Packet Inter-arrival time & $0.0625 \mathrm{sec}$. & $0.166667 \mathrm{sec}$ \\
\hline
\end{tabular}

both have a Poisson process distribution. The metric of ETE delay is tracked throughout the entire simulation. In both scenarios the simulation is limited to 45 minutes; a CSAR Execution phase typically lasts 30 to 45 minutes.

For this study, two experiments each with 10 repetitions are simulated. The first experiment, which simulates 5 different routes under high traffic load, has 10 repetitions for each design (scenario 1: $10+10+10+10+10=50$ ). The second experiment, which simulates 5 different routes under low traffic routes, has 10 repetitions for each design (scenario 2: $10+10+10+10+10=50$ ). The total number of runs for the entire experiment is 100 . For each simulation run, a different random number seed is used.

\section{$\underline{\text { Results }}$}

The data analysis supports half the goals of this research. It supports the high traffic assertion, but it does not support the low traffic hypotheses. In high traffic simulations, ETE delay with the NTO knowledge is shorter than without the NTO. On the other hand, in low traffic simulations, the
ETE delay with the NTO is not shorter than without NTO. Each experiment is replicated 10 times, as stated above, to get a better depiction of the system's performance in terms of ETE delay. Confidence intervals of the $45^{\text {th }}$ minute snapshot are used to evaluate the performance of the system with NTO knowledge in terms of ETE delay.

The ETE delays of two scenarios tested are analyzed by two means. First, the overall ETE delay of the tested scenario is presented. Second, the confidence interval of $45^{\text {th }}$ minute snapshot ETE delays is evaluated. Finally ANOVA is performed in order to show that the variance in performance is a real difference in the changing factor instead of experimental errors.

\section{Analysis of Scenario 1 and 2}

Scenarios 1 and 2 represent the situation with NTO knowledge where 4 different routes are designated to relay the image update or to flood network under high and low traffic load. The overall ETE delay for scenarios 1 and 2 is shown in Figure 6 and Figure 7, respectively. Route 1 indicates the ETE delay without the use of an NTO. Routes 2 through 5 shows the ETE delay with an NTO. By visual inspection from Figure 6, the ETE delay for Routes 2 and 3 and Routes 4 and 5 overlap.

This gives an idea of the feasibility of using the NTO under high traffic load. As expected, ETE delay is shorter with NTO designated routes under high traffic load. By inspecting Figure 6, it can be seen that NTO routes have statistical differences compared to the without NTO route. This difference is shown on the detailed plot containing confidence intervals of $45^{\text {th }}$ minute snapshot in Figure 7.

The validity of the ANOVA can be confirmed by inspecting the residual plots in Figure 8. The normal probability plot and the histogram show the residuals reasonably fit a normal distribution with a mean of zero. The versus fits plot shows residuals evenly distributed above and below the center line with no apparent trends confirming the errors are independent.

\section{Analysis of Scenario 2}

The overall ETE delay for scenario 2 is shown in Figure 9. Route 1 indicates the ETE delay for without the NTO. Routes 2 through 5 show the ETE delay with the NTO. By visual inspection, the ETE delay for Routes 2 and 3 overlap each other, also the ETE delay for Route 1 is shorter when compared to Routes 2 through 5. 


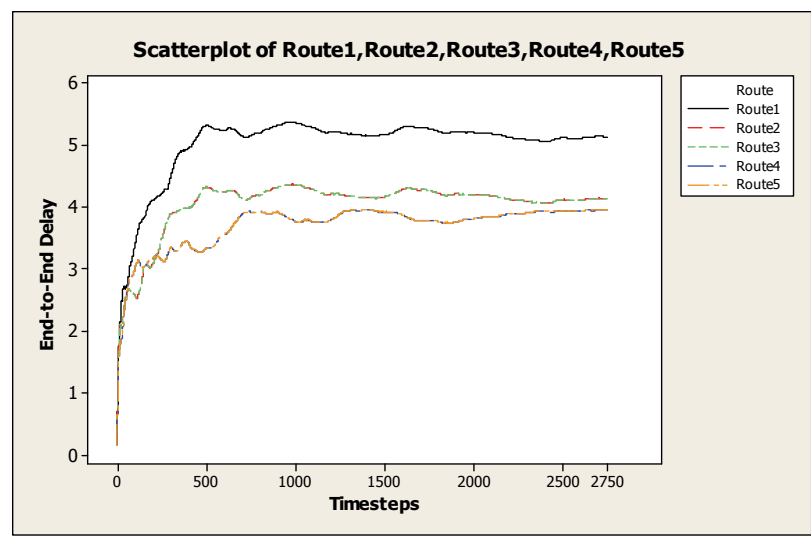

Figure 6. Overall ETE delay for Scenario 1

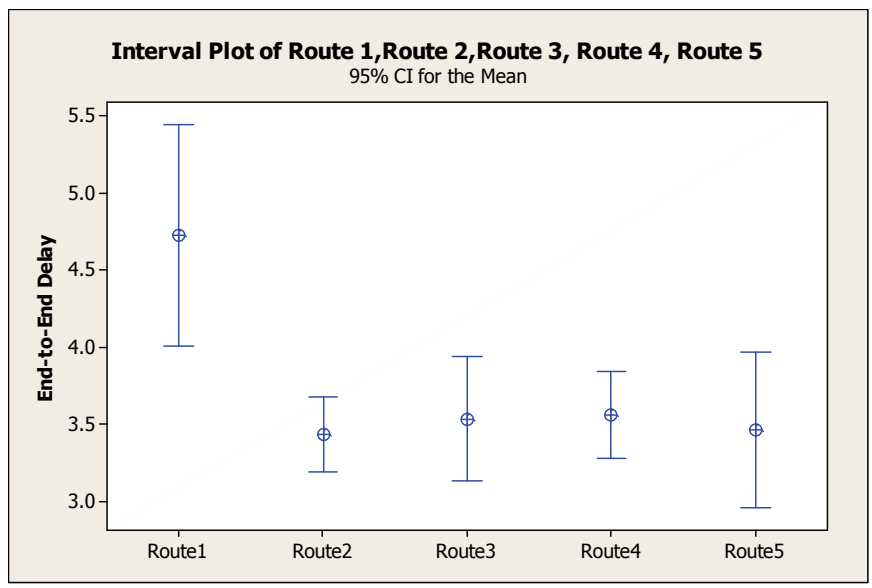

Figure 7. 95\% CI for ETE Delay under High Traffic Load

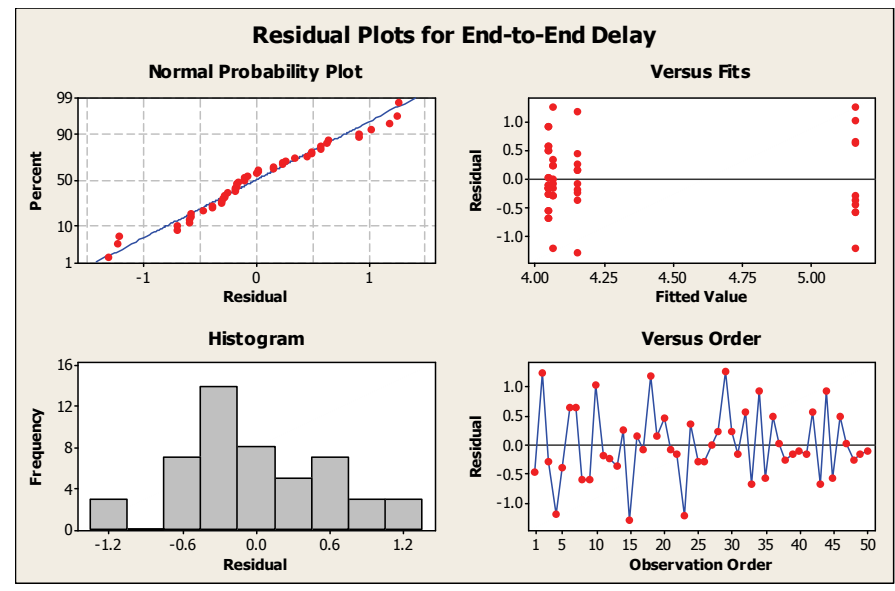

Figure 8. Plots for Verifying the Assumptions of the ETE Delay ANOVA

The ETE delay with an NTO is expected to be shorter compared to the one without an NTO, although the results show that ETE delays are shorter without an NTO under low traffic load. By visual inspection from Figure 9, the route without an NTO has significant statistical difference compared to the one with an NTO. These differences are verified by the numerical data, which is shown on a detailed plot containing confidence intervals in Figure 10.
The validity of the ANOVA can be confirmed by visually inspecting the residual plots in Figure 11.

\section{Overall Analysis}

Statistical analysis confirms the first hypothesis that ETE delay is longer without NTO knowledge in high background traffic. However, the second hypothesis that ETE delay will be longer without the NTO in low traffic, cannot be confirmed with the results of scenario 2 . The NTO knowledge ETE delay under high traffic load provides statistically significant performance gains over the one without an NTO. Using the data from scenario 1, the following performance gain is achieved by the CSAR with NTO routes compared to those without an NTO:

- $19.5 \%$ less ETE delay time via Route 2

- $21.1 \%$ less ETE delay time via Route 3

- $21.5 \%$ less ETE delay time via Route 4

- $21.4 \%$ less ETE delay time via Route 5

Based on scenario 2, the following summarizes the performance achieved with an NTO compared to without:

- $12.2 \%$ higher ETE delay time via Route 2

- $12.8 \%$ higher ETE delay time via Route 3

- $\quad 14.7 \%$ higher ETE delay time via Route 4

- $3.9 \%$ higher ETE delay time via Route 5

There is a big difference in ETE delays under high and low traffic loads. It appears that an NTO provides no benefit in scenario 2. When we look at ETE delays, the NTO runs perform poorly ranging from $3.9 \%$ to $14.7 \%$ worse compared to those without an NTO under low traffic load, but in real life the network will likely never be lightly loaded. In scenario 2, the tradeoff is between bandwidth and speed. Without an NTO you get shorter ETE delays ranging from $3.9 \%$ to $14.7 \%$, but use more bandwidth.

Since the ETE delay with an NTO under high traffic load is less than without an NTO, the comparison between scenarios 1 and 2 can be seen from different aspects. The gain of the first scenario is on average $20.9 \%$. The second scenario loss averages $10.9 \%$. Under low traffic load, if the data is time-critical then flooding is the best option.

\section{Conclusions}

These experiments show that the NTO can be applied to the air assets in the ATO. Since the ATO is highly preplanned, its use can result in lower ETE delay. Decision-makers can gain insight into the network coverage of the air space with the aid of NTO knowledge. Additionally, the results determine that using an NTO significantly outperforms network use without an NTO in terms of reducing ETE delay under high traffic load. 


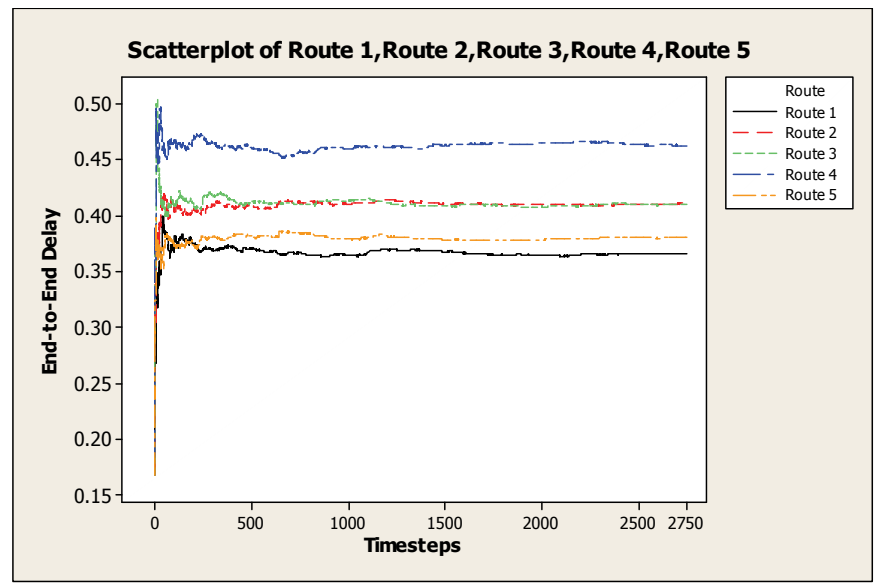

Figure 9. Overall ETE Delay for Scenario 2

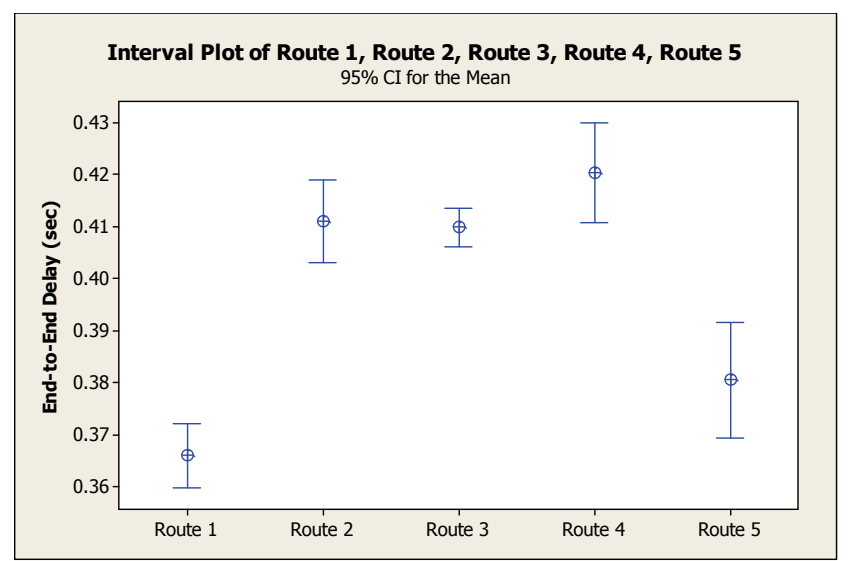

Figure 10. 95\% CI for ETE Delay under Low Traffic Load

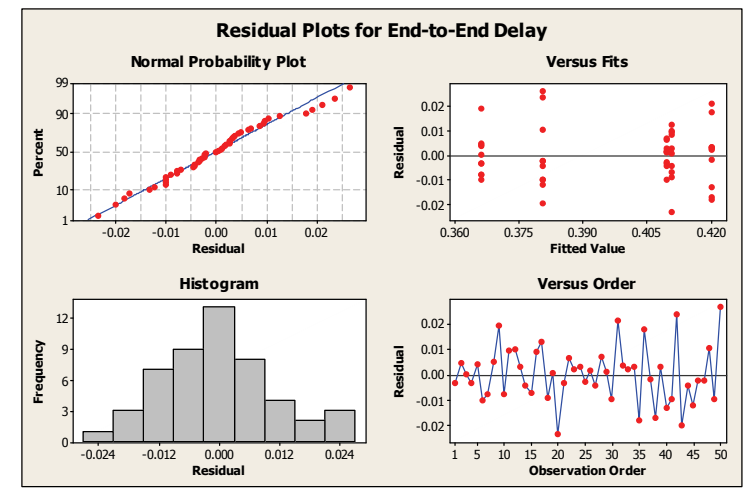

Figure 11. Plots for Verifying the Assumptions of the ETE Delay ANOVA

From the low traffic load perspective, the ETE delay did not outperform the high traffic load, as was expected. This is perhaps due to the fact that flooding causes highly congested links in high traffic conditions and so queuing delay dominates. In low traffic conditions, propagation delay has a higher impact on communication delays, and so flooding is helpful since it inherently chooses the shortest-path route (in addition to also using other redundant routes at the same time).
However, the overall gain can be summarized as follows. Assuming that critical data will be sent under low traffic load, the options will be either flooding the network or choosing routes designated by an NTO. The first option, flooding the network, will give shorter ETE delay, more bandwidth usage, and almost 99\% guaranteed delivery. The second option, choosing the routes designated by an NTO, will give longer ETE delay, less bandwidth usage, and a lower delivery percentage than flooding. We conclude that important traffic should be sent using flooding under low traffic loads.

\section{SUMMARY}

This article advocates the use of an NTO to achieve higher network reliability. In order to shape the FCS, every asset using JTRS must be able to react to rapid changes in the battlefield. By taking advantage of the highly preplanned network coverage in an NTO, reliable and high quality transfers can be achieved. In this article we have shown the benefits of the NTO in the context of CSAR missions.

\section{REFERENCES}

[1] Headquarters Allied Air Forces North, "Air North Manual," NATO.

[2] Department of the Army, "Utility and Cargo Helicopter Operations." vol. Field Manual No. 1-113, June 25, 1997.

[3] J. Kostek, "Harris Corporation Demonstrates Four-Channel Vehicular Radio System," in Communication Solutions, October 6, 2008.

[4] http://www.defenseindustrydaily.com/JTRSAirborne-Maritime-Awards-04833/, "JTRS: Airborne and Maritime Awards," in Defense Industry Daily Magazine, April 6, 2008.

[5] A. Feickert, "The Army's Future Combat System (FCS): Background and Issues for Congress," CRS Report, Ed., November 17, 2005, pp. 1-39.

[6] D. E. Stookey, "A Notional Battlespace for Simulating and Testing Dynamic Wireless Networks," in Electrical and Computer Engineering. vol. Master of Science, WrightPatterson AFB, Ohio: Air Force Institute of Technology, 2006, p. 88.

[7] M. Compton, K. Hopkinson, and S. Graham, "The Network Tasking Order (NTO)," in IEEE Military Communications Conference, San Diego, CA, USA, 2008, pp. 1-7. 